\title{
Giant Fluctuations of Coulomb Drag in a Bilayer System
}

\author{
A. S. Price,${ }^{1}$ A. K. Savchenko, ${ }^{1 *}$ B. N. Narozhny, ${ }^{2}$ G. Allison, ${ }^{1}$ D. A. Ritchie ${ }^{3}$ \\ ${ }^{1}$ School of Physics, University of Exeter, Stocker Road, Exeter, EX4 4QL, UK \\ ${ }^{2}$ The Abdus Salam ICTP, Strada Costiera 11, Trieste I-34100, Italy \\ ${ }^{3}$ Cavendish Laboratory, University of Cambridge, Madingley Road, Cambridge CB3 OHE, UK \\ *To whom correspondence should be addressed: a.k.savchenko@ex.ac.uk.
}

The Coulomb drag in a system of two parallel layers is the result of electronelectron interactions between the layers. We have observed reproducible fluctuations of the drag, both as a function of magnetic field and electron concentration, which are a manifestation of quantum interference of electrons in the layers. At low temperatures the fluctuations exceed the average drag, giving rise to random changes of the sign of the drag. The fluctuations are found to be much larger than previously expected, and we propose a model which explains their enhancement by considering fluctuations of local electron properties.

In conventional measurements of the resistance of a two-dimensional (2D) layer an electrical current is driven through the layer and the voltage drop along the layer is measured. In contrast, Coulomb drag studies are performed on two closely spaced but electrically isolated layers, where a current $I_{1}$ is driven through one of the layers (active layer) and the voltage drop $V_{2}$ is measured along the other (passive) layer (Fig. 1). The origin of this voltage is electron-electron $(e-e)$ interaction between the layers, which creates a 'frictional' force that drags electrons in 
the second layer. The ratio of this voltage to the driving current $R_{D}=-V_{2} / I_{1}$ (the drag resistance) is a measure of $e-e$ interaction between the layers. The measurement of Coulomb drag in systems of parallel layers was first proposed in Ref. (1, 2) and later realised in a number of experiments (3, 4, 5, 6, 7) (for a review see Ref. (8)). As Coulomb drag originates from $e-e$ interactions, it has become a sensitive tool for their study in many problems of contemporary condensed matter physics. For example, Coulomb drag has been used in the search for Bosecondensation of interlayer excitons (9), the metal-insulator transition in two-dimensional (2D) layers (10), and Wigner crystal formation in quantum wires (11).

Electron-electron scattering, and the resulting momentum transfer between the layers, usually creates a so-called 'positive' Coulomb drag, where electrons moving in the active layer drag electrons in the passive layer in the same direction. There are also some cases where unusual, 'negative' Coulomb drag is observed: e.g. between 2D layers in the presence of a strong, quantising magnetic field (6, 7); and between two dilute, one-dimensional wires where electrons are arranged into a Wigner crystal (11). All previous studies of the Coulomb drag, however, refer to the macroscopic (average) drag resistance. Recently there have been theoretical predictions of the possibility to observe random fluctuations of the Coulomb drag (12.13), where the sign of the frictional force will change randomly from positive to negative when either the carrier concentration, $n$, or applied (very small) magnetic field, $B$, are varied.

Drag fluctuations originate from the wave nature of electrons and the presence of disorder (impurities) in the layers. Electrons travel around each layer and interfere with each other, after collisions with impurities, over the characteristic area $\sim L_{\varphi}^{2}$, where $L_{\varphi}$ is the coherence length (Fig. 1). This interference is very important for conductive properties of electron waves. For example, the interference pattern is changed when the phase of electron waves is varied by a small magnetic field, producing universal conductance fluctuations (UCF) seen in small samples with size $L \sim L_{\varphi}$. There is, however, a significant difference between UCF and the fluctuations 
of the drag resistance. The former are only a small correction to the average value of the conductance: in our experiment the single-layer resistance fluctuates by $\sim 200 \mathrm{mOhm}$ around an average resistance of approximately $500 \mathrm{Ohm}$. In contrast, the drag fluctuations, although small in absolute magnitude $(\sim 20 \mathrm{mOhm})$ are able to change randomly, but reproducibly the sign of the Coulomb drag between positive and negative. Surprisingly, we have found that these fluctuations of the Coulomb drag, observed at temperatures below $1 \mathrm{~K}$, are four orders of magnitude larger than predicted in Ref. (12).

Our explanation of the giant drag fluctuations takes into account that, unlike the UCF, the drag fluctuations are not only an interference but also fundamentally an interaction effect. In conventional drag structures the electron mean free path $l$ is much larger than the separation $d$ between the layers, and therefore large momentum transfers $\hbar q$ between electrons in the layers become essential. According to the quantum mechanical uncertainty principle, $\Delta r \Delta q \sim 1$, electrons interact over small distances $\Delta r \ll l$ when exchanging large values of momentum (Fig. 1). As a result the local properties of the layers, such as the local density of electron states $(\mathrm{LDoS})$, become important in the interlayer $e$-e interaction. These local properties at the scale $\Delta r \ll l$ exhibit strong fluctuations (14) that directly manifest themselves in the fluctuations of the Coulomb drag.

The samples used in this work are AlGaAs-GaAs double-layer structures, in which the carrier concentration of each layer can be independently controlled by gate voltage. The two GaAs quantum wells of the structure, $200 \AA$ in thickness, are separated by an $\mathrm{Al}_{0.33} \mathrm{Ga}_{0.67} \mathrm{As}$ layer of thickness $300 \AA$. Each layer has a Hall-bar geometry, $60 \mu \mathrm{m}$ in width and with a distance between the voltage probes of $60 \mu \mathrm{m}(15)$.

Figure 2 shows the appearance of the fluctuations in the drag resistivity, $\rho_{D}$, at low temperatures. At higher temperatures, the drag resistance changes monotonically with both $T$ and $n$ : the insets to Fig. 2 show that $\rho_{D}$ increases with increasing temperature as $T^{2}$ and decreases 
with increasing passive-layer carrier concentration as $n_{2}^{b}$, where $b \approx-1.5$. These results are consistent with existing experimental work on the average Coulomb drag (4 16$)$.

Figure 3A shows a zoomed-in view of the reproducible fluctuations as a function of $n_{2}$. These fluctuations result in an alternating sign of the drag, which is demonstrated in the inset to Fig. 3 where the temperature dependence of the drag is shown at two different values of $n_{2}$. The drag is seen first to decrease as the temperature is decreased, but then become either increasingly positive or increasingly negative, dependent upon $n_{2}$. The reproducible fluctuations of the drag resistivity have also been observed as a function of magnetic field (Fig. 3B). For a fixed temperature, the magnitude of the drag fluctuations as a function of $n_{2}$ is roughly the same as that as a function of $B$.

The theory of Ref. (12) calculates the variance of drag fluctuations in the so-called diffusive regime, $l<d$. In this case the drag is determined by global properties of the layers, averaged over a region $\Delta r \gg l$. The expected variance of drag fluctuations (at low $T$ when the fluctuations exceed the average) in the diffusive regime is

$$
\left\langle\Delta \sigma_{D}^{2}\right\rangle \approx A \frac{e^{4}}{\hbar^{2}} \frac{E_{T}(L) \tau_{\varphi} \ln \kappa d}{g^{4} \hbar(\kappa d)^{3}},
$$

where $\sigma_{D} \approx \rho_{D} /\left(\rho_{1} \rho_{2}\right)$, and $\rho_{1}$ and $\rho_{2}$ are the active and passive layer resistivities, respectively; $E_{T}(L)$ is the Thouless energy, $E_{T}(L)=\hbar D / L^{2}, D$ is the diffusion coefficient; $\tau_{\varphi}$ is the decoherence time; $\kappa$ is the inverse screening length; $A=4.9 \times 10^{-3}$ and $g=h /\left(e^{2} \rho\right)$ is the dimensionless conductivity of the layers. Using the parameters of our system, this expression gives a variance of $\sim 6 \times 10^{-11} \mu \mathrm{S}^{2}$, which is approximately eight orders of magnitude smaller than the variance of the observed drag fluctuations. The fluctuations in $\rho_{D}$ have been measured in two different samples, and their variance is seen to be similar in magnitude and $T$-dependence, confirming the discrepancy with the theoretical prediction (12).

The expected fluctuations of the drag conductivity share the same origin as the UCF in 
the conventional conductivity: coherent electron transport over $L_{\varphi}$ in the layers prior to $e-e$ interaction between the layers (Fig. 1). For this reason we have compared the drag fluctuations with the fluctuations seen in the single-layer resistivity of the same structure (Fig. 3B, inset), which have shown the usual behaviour (17). We estimate the expected variance of the singlelayer conductance fluctuations using the relation $\left\langle\Delta \sigma_{x x}^{2}\right\rangle=\left(e^{2} / h\right)^{2}\left(L_{T} / L\right)^{2}$, where $L_{T}=$ $\sqrt{\hbar D / k_{B} T}$ is the thermal length (17). This expression produces a value of $0.8 \mu \mathrm{S}^{2}$, which is in good agreement with the measured value of $0.6 \mu \mathrm{S}^{2}$. The typical 'period' of the drag fluctuations (the correlation field, $\Delta B_{c}$ ) is similar to that of the UCF (15), indicating that both depend upon the same $L_{\varphi}$ and have the same quantum origin.

To address the question of the discrepancy between the magnitude of drag fluctuations in theory (12) and our observations, we stress that the theoretical prediction for the variance, Eq.11, was obtained under the assumption of diffusive motion of interacting electrons, with small interlayer momentum transfers, $q \ll 1 / l$. As the layers are separated by a distance $d$, the $e-e$ interactions are screened at distances $\Delta r>d$. Therefore, in all regimes the maximum momentum transfers are limited by $q<1 / d$. In the diffusive regime, $l<d$, this relation also means that $q<1 / l$, that is, interlayer $e$-e interactions occur at distances $\Delta r>l$ and involve scattering by many impurities in the individual layers. In the opposite situation, $l \gg d$, the transferred momenta will include both small and large $q$-values: $q<1 / l$ and $1 / l<q<1 / d$. We have seen that small $q$ cannot explain the large fluctuations of the drag (12), and so argue that it is large momentum transfers with $q>1 / l$ which give rise to the observed effect. In this case the two electrons interact at a distance $\Delta r$ that is smaller than the average impurity separation and, therefore, it is the local electron properties of the layers which determine $e$-e interaction. In Ref. (14) it is shown that the fluctuations of the local properties are larger compared to those of the global properties that are responsible for the drag in the diffusive case.

A theoretical expression for the drag conductivity is obtained by means of a Kubo formula 
analysis (18, 19,20, 21) (detailed description in supporting text). For a qualitative estimate, three factors have to be taken into account: (i) the inter-layer matrix elements of the Coulomb interaction $\mathcal{D}_{i j}$; (ii) the phase space (the number of electron states available for scattering); and (iii) the electron-hole $(e-h)$ asymmetry in both layers. Point (iii) takes into account that in a quantum system the current is carried by both electron-like (above the Fermi surface) and holelike (below the Fermi surface) excitations. If they were completely symmetric with respect to each other, then the current-carrying state of the active layer would have zero total momentum and thus no drag effect would be possible. The physical quantity that measures the degree of $e-h$ asymmetry is the non-linear susceptibility $\Gamma$ of the $2 \mathrm{D}$ layer. Theoretically, the drag conductivity is represented in terms of the non-linear susceptibilities of each layer and dynamically screened interlayer Coulomb interaction $\mathcal{D}_{i j}(\omega)$ as $\sigma_{D} \propto \int d \omega \mathcal{D}_{12}(\omega) \Gamma_{2}(\omega) \mathcal{D}_{21}(\omega) \Gamma_{1}(\omega)$ (indices 1 and 2 correspond to the two layers) (18, 12). The $e$-h asymmetry appears in $\Gamma$ as a derivative of the density of states $\nu$ and the diffusion coefficient $D: \Gamma \propto \partial(\nu D) / \partial \mu$, and it is this quantity that is responsible for the fact that drag fluctuations can exceed the average. As $D \nu \sim g$ and the typical energy of electrons is the Fermi energy, $E_{F}$, we have $\partial(\nu D) / \partial \mu \sim g / E_{F}$ for the average drag. The typical energy scale for the interfering electrons, however, is $E_{T}\left(L_{\varphi}\right)$ (17), which is much smaller than $E_{F}$ and therefore a mesoscopic system has larger $e$ - $h$ asymmetry.

Under the condition of large momentum transfer between the layers, fluctuations in $\Gamma$ are similar to the fluctuations of the $\mathrm{LDoS}$, which can be estimated as $\delta \nu^{2} \sim\left(\nu^{2} / g\right) \ln \left(\max \left(L_{\varphi}, L_{T}\right) / l\right)$ (14). Also, the interaction in the ballistic regime can be assumed to be constant, $\mathcal{D}_{i j} \approx-1 / \nu \kappa d$, as $q$ is limited by $q \leq 1 / d$. Finally, to average fluctuations of the drag over the sample with size $L$ we should divide it into coherent patches of size $L_{\varphi}$ that fluctuate independently and thus decrease the total variance: $\left\langle\Delta \sigma_{D}^{2}\right\rangle=\left\langle\Delta \sigma_{D}^{2}\left(L_{\varphi}\right)\right\rangle\left(L_{\varphi} / L\right)^{2}$. If $k_{B} T>E_{T}\left(L_{\varphi}\right)$, fluctuations are further averaged on the scale of $\sim k_{B} T$, and therefore the variance is suppressed by an additional factor of $E_{T}\left(L_{\varphi}\right) / k_{B} T$. Combining the above arguments we find 


$$
\left\langle\Delta \sigma_{D}^{2}\right\rangle=N \frac{e^{4}}{g^{2} \hbar^{2}(\kappa d)^{4}} \frac{\left(k_{B} T\right)^{2}}{E_{T}^{2}\left(L_{\varphi}\right)} \frac{l^{4} L_{\varphi}^{2}}{d^{4} L^{2}},
$$

where $N$ is a numerical coefficient.

Compared to the diffusive situation (Eq. 1) the fluctuations described by our model are greatly enhanced. The difference between Eqs. 2] and 1 comes from the fact that in the ballistic regime electrons are not scattered by impurities between events of $e$ - $e$ scattering. Large momentum transfers correspond to short distances, and thus in the ballistic regime drag measurements explore the local (as opposed to averaged over the whole sample) non-linear susceptibility. This leads to the appearance of three extra factors in Eq. 2; (i) the factor $l^{4} / d^{4}$ (which is also present in the average drag in the ballistic regime - see Ref. (18)); (ii) the phase space factor $T / E_{T}$ (which appears due to the fact that interaction parameters $\mathcal{D}_{i j}$ are now energy-independent); and (iii) the extra factor $g^{2}$ due to fluctuations of the local non-linear susceptibility. Local fluctuations are enhanced since the random quantity $\Gamma$ is now averaged over a small part of the ensemble, allowing one to detect rare impurity configurations.

Our model not only explains the large magnitude of the fluctuations, but also predicts a non-trivial temperature dependence of their magnitude. The latter comes from the change in the temperature dependence of $L_{\varphi}(22)$ : at low temperatures, $k_{B} T \tau / \hbar \ll 1$, the usual result is $L_{\varphi} \propto T^{-1 / 2}$, while for $k_{B} T \tau / \hbar>1$ the temperature dependence changes to $L_{\varphi} \propto T^{-1}(23)$. Consequently, the temperature dependence of the variance of the drag fluctuations is expected to change from $T^{-1}$ at low $T$, to $T^{-4}$ at high $T$. This temperature dependence is very different from the $T$-dependence of drag fluctuations in the diffusive regime, $\left\langle\Delta \sigma_{D}^{2}\right\rangle \propto T^{-1}$. To test the prediction of Eq. 2, the $T$-dependence of $\left\langle\Delta \sigma_{D}^{2}\right\rangle$ has been analysed (Fig. 4). The variance is calculated in the limits of both the diffusive $\tau_{\varphi}$ ( solid line, $\tau_{\varphi}^{-1} \propto T$ ) and ballistic $\tau_{\varphi}$ (dashed line, $\tau_{\varphi}^{-1} \propto T^{2}$ ), using $N \simeq 10^{-4}$. In fitting the drag variance we have found $\tau_{\varphi}$ to agree with theory to within a factor of two $(15)$, which is typical of the agreement found in other 
experiments on determining $\tau_{\varphi}$ (24). (The single-layer values of $\tau_{\varphi}$ found from our analysis of the UCF agree with theory to within a factor of 1.5.) Thus, the temperature dependence of the observed drag fluctuations strongly supports the validity of our explanation.

We have observed reproducible fluctuations of the Coulomb drag and demonstrated that they are an informative tool for studying wave properties of electrons in disordered materials, and the local properties in particular. Contrary to UCF which originate from quantum interference, fluctuations of drag result from an interplay of the interference and $e-e$ interactions. More theoretical and experimental work is required to study their manifestation in different situations. For instance, similarly to the previous extensive studies of the evolution of UCF with increasing magnetic field, such experiments can be performed on the fluctuations of drag. One of the important developments in the field of Coulomb drag fluctuations can be their study in quantising magnetic fields, including the regimes of integer and fractional quantum Hall effects. 


\section{References}

1. M. B. Pogrebinskii, Sov. Phys. Semicond. 11, 372 (1977).

2. P. J. Price, Physica 117B, 750 (1983).

3. P. M. Solomon, P. J. Price, D. J. Frank, D. C. La Tulipe, Phys. Rev. Lett. 63, 2508 (1989).

4. T. J. Gramila, J. P. Eisenstein, A. H. MacDonald, L. N. Pfeiffer, K. W. West, Phys. Rev. Lett. 66, 1216 (1991).

5. U. Sivan, P. M. Solomon, H. Shtrikman, Phys. Rev. Lett. 68, 1196 (1992).

6. M. P. Lilly, J. P. Eisenstein, L. N. Pfeiffer, K. W. West, Phys. Rev. Lett. 80, 1714 (1998).

7. J. G. S. Lok et al., Phys. Rev. B 63, 041305 (2001).

8. A. G. Rojo, J. Phys.: Condens. Matter 11, R31 (1999).

9. D. Snoke, Science 298, 1368 (2002).

10. R. Pillarisetty et al., Physical Review B 71, 115307 (2005).

11. M. Yamamoto, M. Stopa, Y. Tokura, Y. Hirayama, S. Tarucha, Science 313, 204 (2006).

12. B. N. Narozhny, I. L. Aleiner, Phys. Rev. Lett. 84, 5383 (2000).

13. N. A. Mortensen, K. Flensberg, A.-P. Jauho, Phys. Rev. B 65, 085317 (2002).

14. I. V. Lerner, Phys. Lett. A 133, 253 (1988).

15. The details of the structures, measurements and the model are available as supporting material on Science Online. 
16. M. Kellogg, J. P. Eisenstein, L. N. Pfeiffer, K. W. West, Solid State Communications 123, 515 (2002).

17. B. L. Altshuler, P. A. Lee, R. A. Webb, eds., Mesoscopic Phenomena in Solids (NorthHolland, New York, 1991).

18. A. Kamenev, Y. Oreg, Phys. Rev. B 52, 7516 (1995).

19. L. Zheng, A. H. MacDonald, Phys. Rev. B 48, 8203 (1993).

20. A.-P. Jauho, H. Smith, Phys. Rev. B 47, 4420 (1993).

21. K. Flensberg, B. Y.-K. Hu, A.-P. Jauho, J. M. Kinaret, Phys. Rev. B 52, 14761 (1995).

22. B. N. Narozhny, G. Zala, I. L. Aleiner, Phys. Rev. B 65, 180202 (2002).

23. B. L. Altshuler, A. G. Aronov, Electron-Electron Interactions in Disordered Systems, A. L. Efros, M. Pollak, eds. (North-Holland, Amsterdam, 1985).

24. C. W. J. Beenakker, H. van Houten, Solid State Physics, H. Ehrenreich, D. Turnbull, eds. (Academic Press Limited, 1991).

25. Authors thank I.L. Aleiner, M. Entin, I.L. Lerner, A. Kamenev, and A. Stern for numerous helpful discussions.

\section{Supporting Online Material}

Www.sciencemag.org

Materials and Methods

SOM text

Figs. S1 to S3 


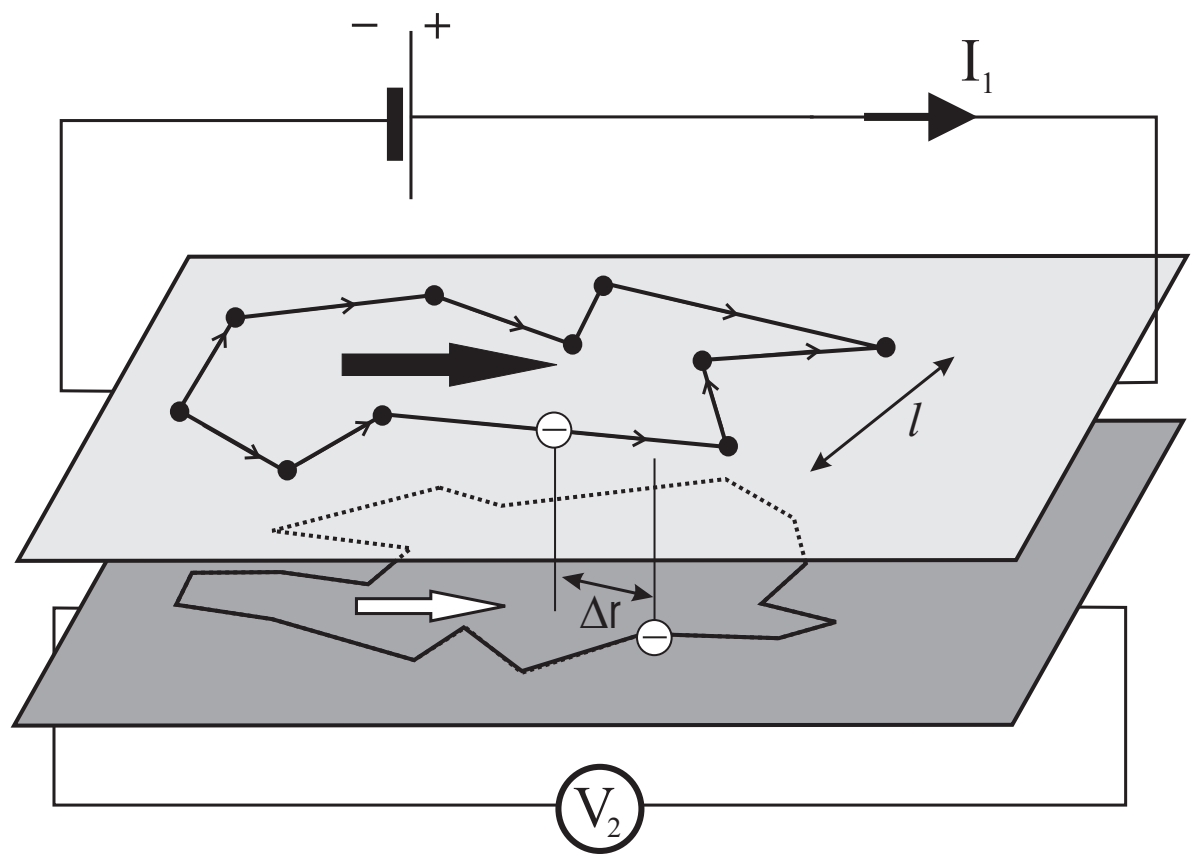

Fig. 1. Schematic showing the origin of the drag signal $V_{2}$ induced by the current $I_{1}$. The fluctuations of the drag arise from the interference of electron waves in each layer, before the two electrons take part in the interlayer interaction. 


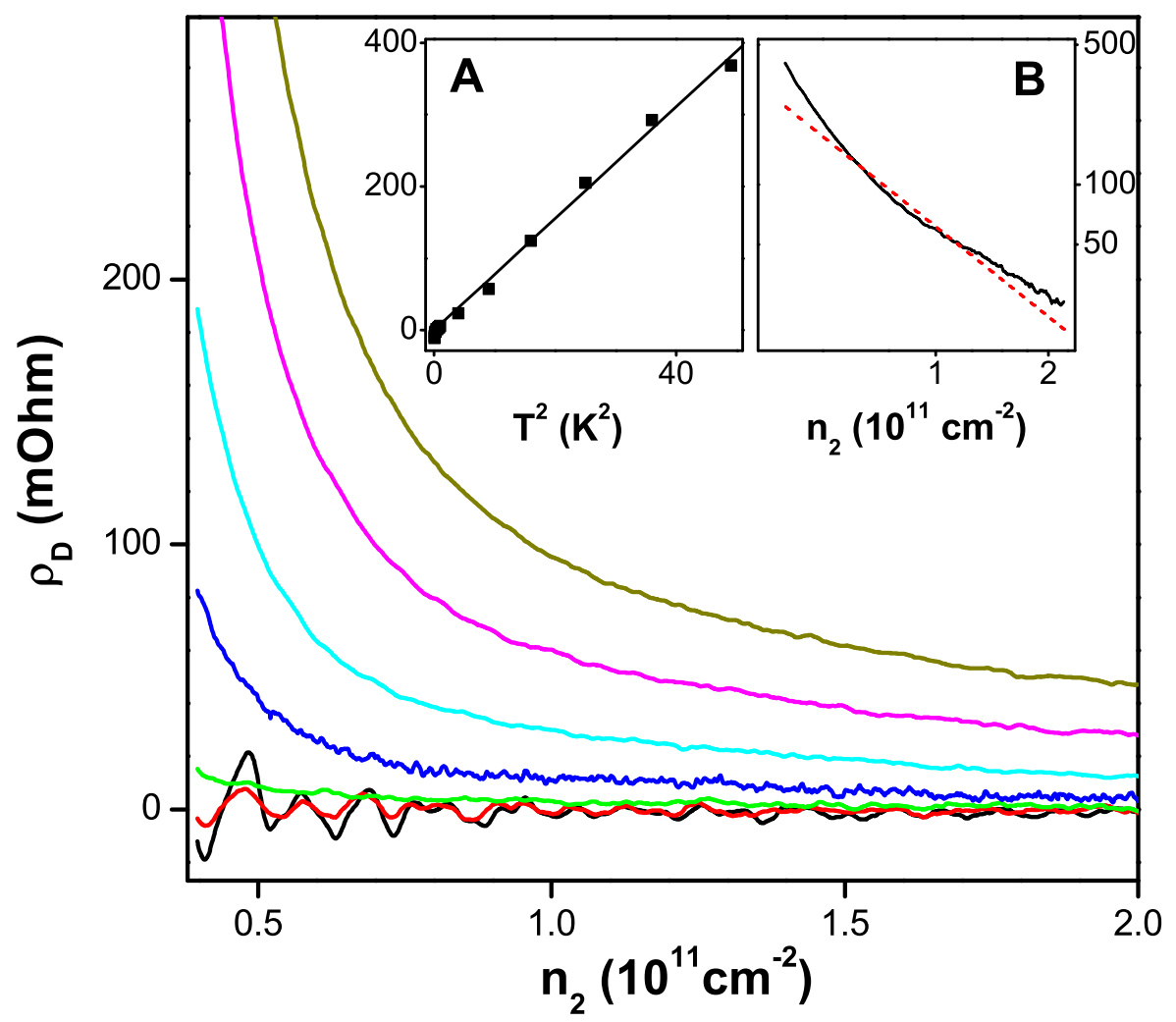

Fig. 2. Drag resistivity as a function of passive-layer carrier concentration for different temperatures: $T=5,4,3,2,1,0.4$, and $0.24 \mathrm{~K}$, from top to bottom. Inset $(\mathbf{A}): \rho_{\mathrm{D}}$ as a function of $T^{2}$. Inset $(B): \rho_{D}$ as a function of $n_{2}$, with $n_{1}=1.1 \times 10^{11} \mathrm{~cm}^{-2}$; dashed line is a $n_{2}^{-1.5}$ fit. 


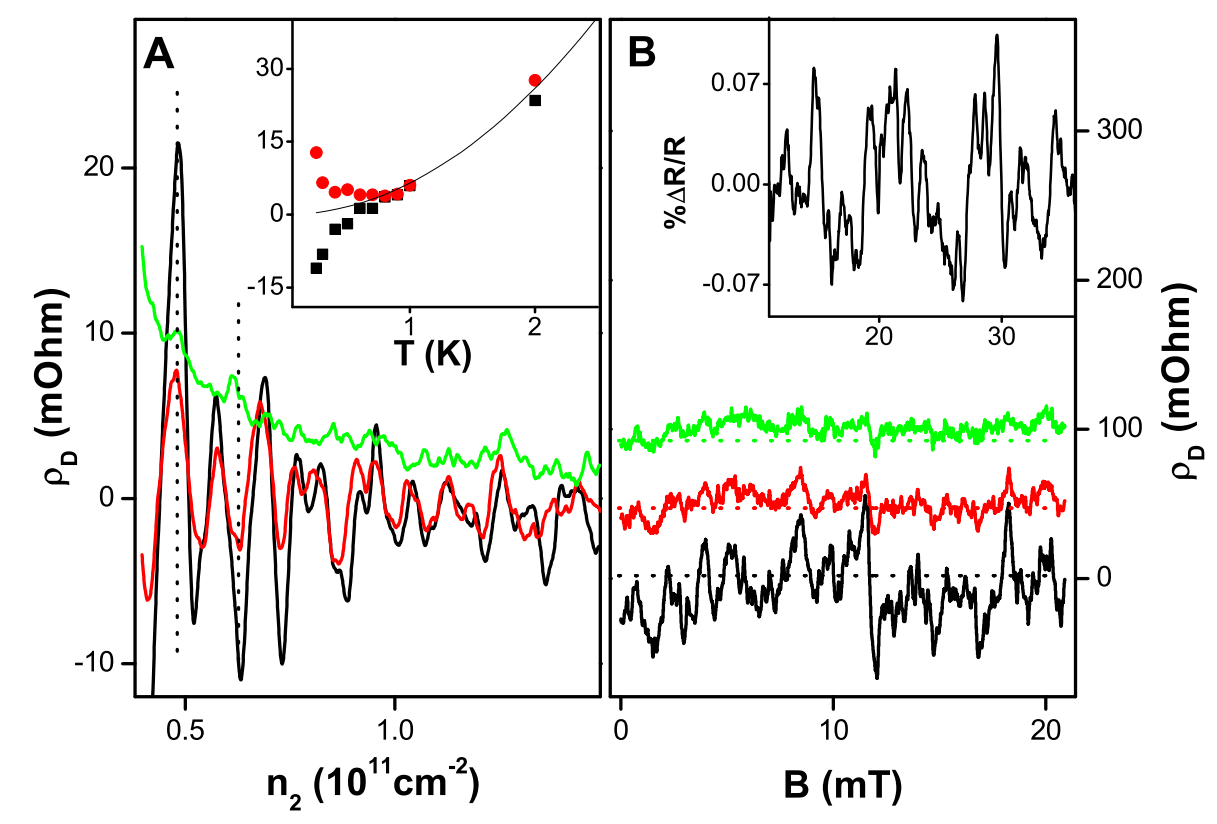

Fig. 3. (A) Drag resistance measured at low temperatures as a function of passivelayer concentration; $T=1,0.4$, and $0.24 \mathrm{~K}$, from top to bottom. Inset: $\rho_{\mathrm{D}}$ as a function of $T$ for two values of $n_{2}$ denoted by the dotted lines in Fig. 3A; solid line is the expected $T^{2}$ dependence of the average drag. $(B): \rho_{D}$ as a function of $B ; T=0.4,0.35$, and 0.24 $\mathrm{K}$, from top to bottom. (Graphs for higher T are vertically offset for clarity.) Single-layer concentration for each layer is $5.8 \times 10^{10} \mathrm{~cm}^{-2}$. Inset: The UCF of the single-layer, with an average background resistance of $500 \mathrm{Ohm}$ subtracted. 


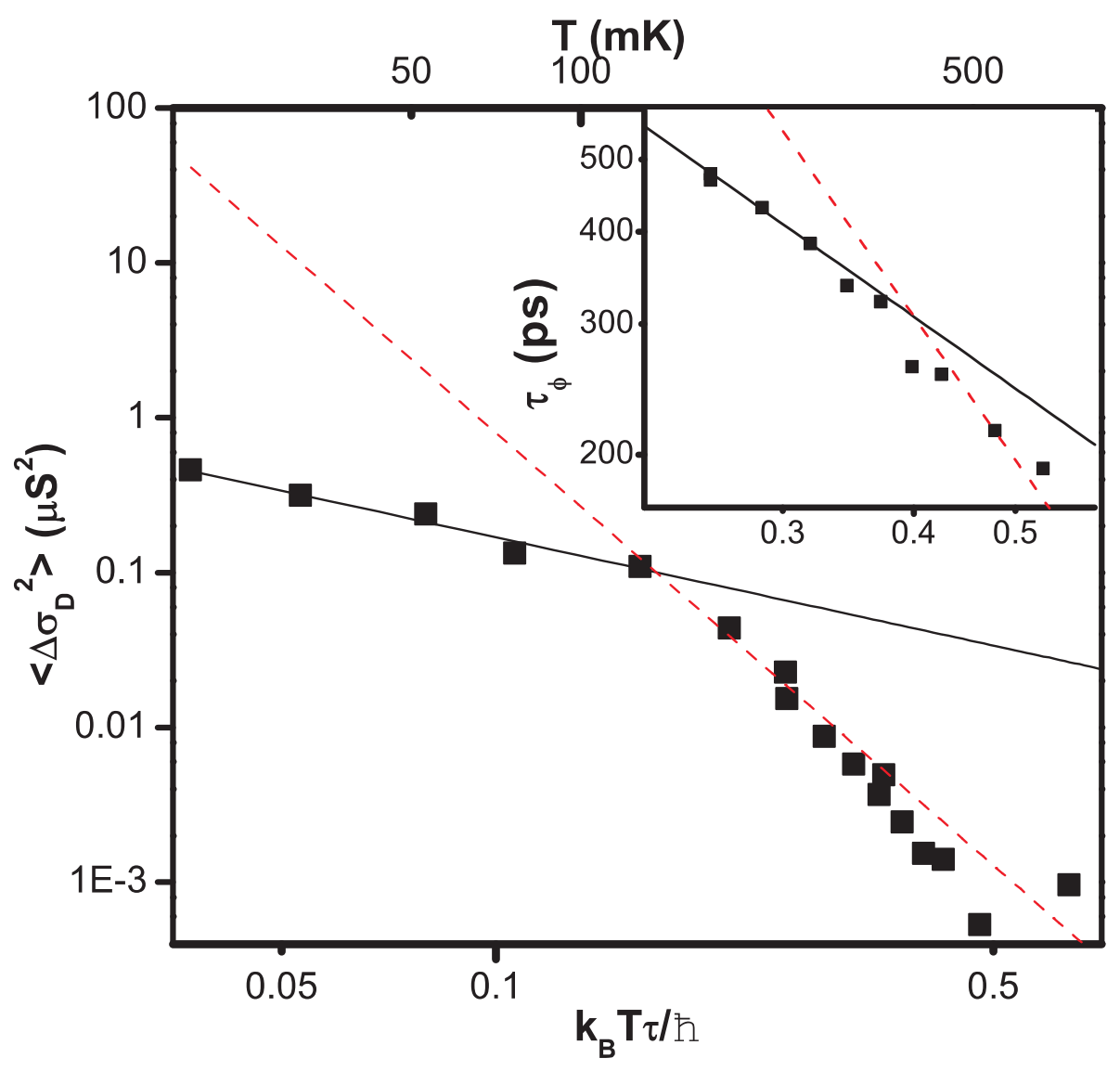

Fig. 4. The variance of the drag conductivity fluctuations (squares) plotted against temperature. The solid and dashed lines are calculated using Eq. 2] with the diffusive and ballistic asymptotes of $\tau_{\varphi}$, respectively. Inset: $\tau_{\varphi}$ extracted from the correlation magnetic field of the single-layer fluctuations, plotted against temperature. 